\title{
Angiostrongylus meningoencephalitis: survival from minimally conscious state to rehabilitation
}

\section{Nicholas F Blair \\ BSc, MB BS, Neurology Registrar \\ Carolyn F Orr MRCP, FRACP, PhD Neurologist ${ }^{2}$

\begin{abstract}
Anthony P Delaney MB BS, MSC, FCICM,
\end{abstract} Intensive Care Physician \\ Geoffrey K Herkes MB BS, PhD, FRACP Staff Specialist ${ }^{3}$ \\ 1 Royal North Shore Hospital, Sydney, NSW. \\ 2 Macquarie University Hospital, Sydney, NSW. \\ 3 Northern Clinical School, University of Sydney, Sydney, NSW. \\ gherkes@nsccahs. health.nsw.gov.au}

doi: 10.5694/mjal2.11085

\begin{abstract}
The nematode Angiostrongylus cantonensis has spread down the eastern coast of Australia over recent decades. A healthy 21-year-old man developed life-threatening eosinophilic meningoencephalitis following ingestion of a slug in Sydney. We describe the first case of this severity in which the patient survived.
\end{abstract}

\section{Clinical record}

A 21-year-old man presented with a 3-day history of insomnia and paraesthesia affecting his lower limbs bilaterally. He had no associated headache, meningism or fever. He was previously well with no significant medical history.

On admission, he had begun to develop progressive weakness of his lower limbs associated with pain and dysaesthesia. A full blood count showed a total white cell count of $10.6 \times 10^{9} / \mathrm{L}$ (reference interval [RI], 4.0-11.0 $\times 10^{9} / \mathrm{L}$ ) with mild eosinophilia $\left(0.5 \times 10^{9} / \mathrm{L}\left[\mathrm{RI},<0.4 \times 10^{9} / \mathrm{L}\right]\right)$. Magnetic resonance imaging (MRI) scans of his brain and spine showed no abnormality. His cerebrospinal fluid (CSF) was acellular, with normal glucose and protein levels.

A provisional diagnosis of Guillain-Barré syndrome was made and the patient was treated with a 5-day course of intravenous immunoglobulin. Over 1 week he developed evidence of autonomic instability with urinary retention, fluctuating sinus tachycardia and hypertension, and a paralytic ileus. By the second week of hospitalisation he had developed hallucinations and a fluctuating level of consciousness.

A repeat CSF sample revealed a raised protein level of $1.20 \mathrm{~g} / \mathrm{L}$ (RI, $0.15-0.45 \mathrm{~g} / \mathrm{L}$ ), a low glucose level of $2.3 \mathrm{mmol} / \mathrm{L}$ (RI, $2.5-5.6 \mathrm{mmol} / \mathrm{L}$ ), with $2 \times 10^{9} / \mathrm{L}$ red cells $\left(\mathrm{RI},<5 \times 10^{9} / \mathrm{L}\right), 406 \times 10^{9} / \mathrm{L}$ mononuclear cells $(\mathrm{RI},<5 \times$ $10^{9} / \mathrm{L}$ ), and $30 \times 10^{9} / \mathrm{L}$ polymorphs (RI, nil). The opening pressure was elevated at $31 \mathrm{~cm} \mathrm{H}_{2} \mathrm{O}\left(\mathrm{RI}, 6-20 \mathrm{~cm} \mathrm{H}_{2} \mathrm{O}\right)$. He was commenced on empirical antibiotic and antiviral treatment, with intravenous hydrocortisone $(100 \mathrm{mg}$ four times daily) to cover the possibility of a steroid-responsive encephalopathy. An electroencephalogram was consistent with generalised encephalopathy without focal epileptiform activity. CSF bacterial cultures, cryptococcal antigen testing and polymerase chain reaction testing for herpes simplex virus and enterovirus were negative. HIV serological testing was negative. The patient's condition continued to deteriorate, with a declining level of consciousness, progressive quadriparesis and respiratory failure necessitating endotracheal intubation and mechanical ventilation on Day 12 after admission.

Progress computed tomography (CT) brain imaging results remained normal. His peripheral eosinophil count had risen, later peaking at $1.9 \times 10^{9} / \mathrm{L}$ on Day 24 . A third lumbar puncture was performed. His CSF protein remained elevated at $0.71 \mathrm{~g} / \mathrm{L}, \mathrm{CSF}$ red cell count was $216 \times$ $10^{9} / \mathrm{L}$ and CSF white cell count was $504 \times 10^{9} / \mathrm{L}$. Specific staining for eosinophils was performed, showing $37 \%$ of the leukocytes to be eosinophils (RI, <10\%, Box 1).

By this stage it had emerged that the patient had eaten a slug from a Sydney garden, as a dare, 7 days before presentation. An enzyme immunoassay for Angiostrongylus IgG performed on the CSF was positive. A progress MRI scan, performed on Day 26 after admission, revealed multiple foci of hyperintensity in the cerebral hemispheres, brainstem and cerebellum as well as within the spinal cord (Box 2). Several of the lesions showed restricted diffusion and some showed contrast enhancement. Pial enhancement was seen within the posterior fossa and over the spinal cord.

Treatment with high-dose corticosteroids was continued but the patient's condition continued to decline. An unresponsive state developed, with flaccid tone in all four limbs and the loss of brainstem reflexes. Given the severity of the patient's condition, a trial of albendazole $400 \mathrm{mg}$ twice daily was given, with continued corticosteroid cover (dexamethasone $4 \mathrm{mg}$ intravenously four times daily) and he

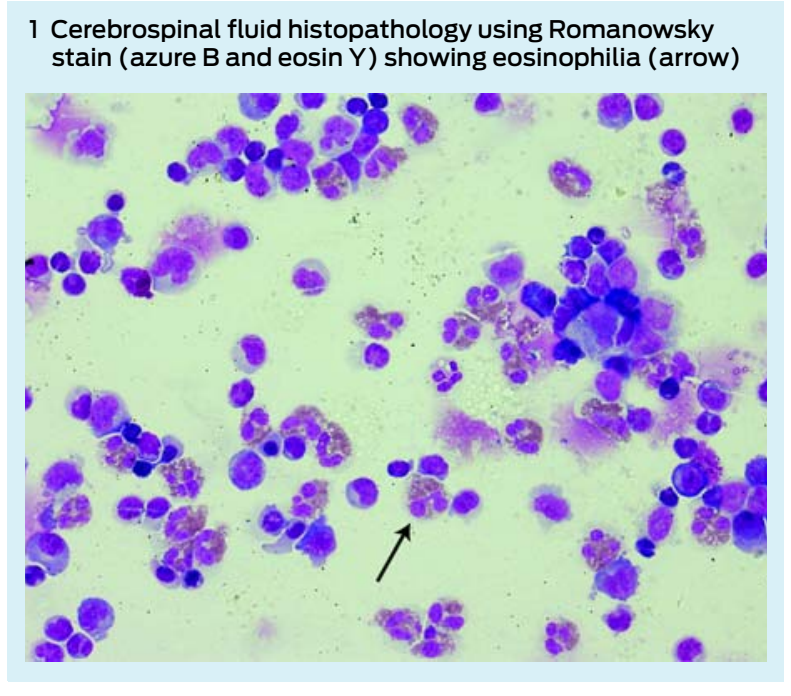


2 Magnetic resonance imaging of the brain showing (A) T2 fluid-attenuated inversion recovery (FLAIR) image with gadolinium contrast, showing multiple small foci of enhancement (arrows), and (B) some lesions showing restricted diffusion on diffusion-weighted imaging (arrows)
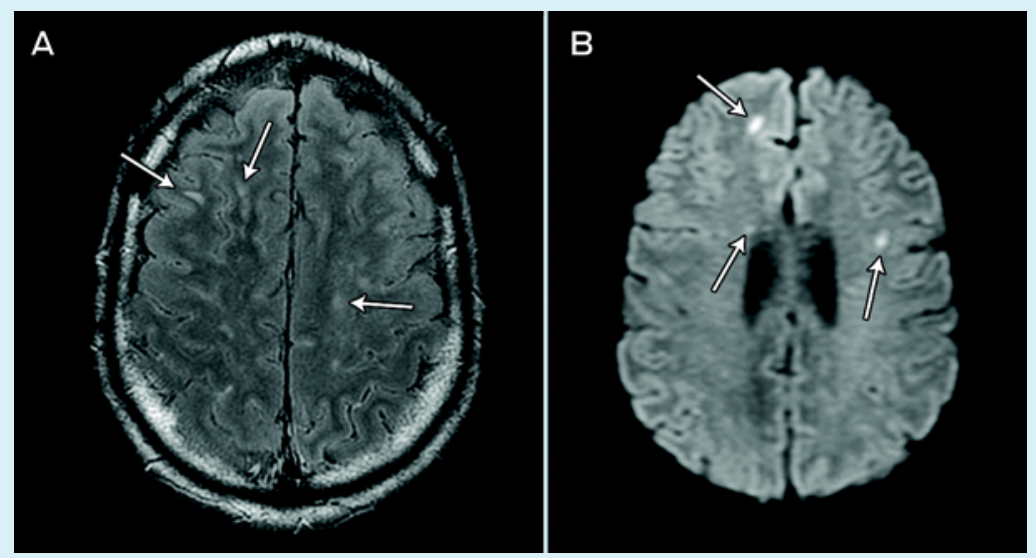

remained on this treatment for 1 month. There was no change in his condition and he remained supported by mechanical ventilation via a tracheostomy in a minimally conscious state for 8 months.

During this time, there was much discussion between the patient's family and treating doctors about his prognosis and probable outcome. Treatment was continued on the basis of his age and the uncertainty of the natural history of this rare disease. His clinical course was complicated by hydrocephalus requiring a ventriculoperitoneal shunt, recurrent episodes of ventilator-associated pneumonia, and seizures that were difficult to control despite multiple antiepileptic drugs. After 13 months, there was a very slow improvement in his level of consciousness, such that a slow weaning of respiratory support could be attempted. He could successfully maintain his own ventilation during the day (though with an ataxic respiratory pattern), but remained dependent on nocturnal mechanical ventilation.

The patient was discharged to the ward from intensive care in the 15th month of admission, where he continued to make slow but definite progress. There was gradual recovery of some distal power in his upper and lower limbs and he developed the ability to communicate with head movements. He was discharged to a rehabilitation facility 22 months after admission, where there has been ongoing gradual improvement. He now has antigravity power in his limbs and is capable of more complex nonverbal communication.

\section{Discussion}

Angiostrongylus cantonensis, also known as the rat lungworm, is the most common cause of eosinophilic meningitis globally. This condition generally follows a benign, selflimited course. ${ }^{1}$ Rarely, the parasite causes meningoencephalitis, which should be considered a related but distinct clinical entity with a dramatically poorer prognosis. The mortality rate has been reported at $79 \%{ }^{2}$ and, of patients who become comatose, at least $90 \%$ do not survive. $^{3}$
A. cantonensis is endemic in South-East Asia and the Pacific region, and has spread down the eastern coast of Australia over the past 50 years. ${ }^{4}$ In Australia, it has been observed that cases tend to be particularly severe. This reflects the higher total larval load ingested from terrestrial hosts, which feed on rat faecal pellets harbouring thousands of larvae. In comparison, aquatic snails, which commonly cause the disease in South-East Asia, generally carry a smaller larval load. ${ }^{5,6}$ The first reported human case acquired in Sydney occurred in 2001, ${ }^{7}$ in the remarkably similar circumstances of a young man accepting a dare to eat a slug, highlighting the importance of specific questioning in the patient's history. Our patient's case is only the second reported case acquired in Sydney and, internationally, our patient is the first with the disease of this severity to have survived.

Recent investigations have sought to identify factors associated with the development of clinically severe angiostrongyliasis. In one study, clinical features including headache, abnormal CSF pressure and abnormal peripheral blood eosinophil count were associated with severe disease. $^{8}$ An Activation Criteria for Angiostrongyliasis (ACA) scoring system, incorporating these factors, was proposed and validated in a population of Chinese patients, with a score of $\geqslant 7$ predictive of severe disease. If we had used the presenting eosinophil count, our patient would only have had an ACA score maximum of 5, and most likely lower than this if his CSF opening pressure had been recorded at the first lumbar puncture. He would have scored 8 if his peak peripheral eosinophil count and his highest recorded CSF pressure had been used.

A second study investigated factors specifically associated with the development of the encephalitic form of the disease. $^{2}$ In a cohort of 94 patients with angiostrongyliasis, of whom 14 developed encephalitis, it was found that the clinical factors predictive of encephalitis were temperature $>38^{\circ} \mathrm{C}$ at presentation, older age and longer duration of headache. Fever at presentation was associated with a remarkable 37-fold risk of encephalitis. Interestingly, other variables such as CSF opening pressure, peripheral or CSF eosinophil counts or paraesthesia were not predictive of encephalitis in this study. Our case indicates that caution should be used when applying the predictive factors reported in these studies, and suggests that peak eosinophil count and delayed CSF pressure results may be more useful when calculating the ACA.

The initial difficulty with diagnosis in this case emphasises the need for clinical suspicion of this condition in the setting of acute-onset neurological symptoms and peripheral eosinophilia in endemic areas, including the eastern coast of Australia. It is essential to seek a history of consuming raw or undercooked food, and specifically any ingestion of molluscs. The case illustrates the importance of repeat CSF examination if the diagnosis is suspected and the initial CSF test results are negative. It also highlights the need to request specific CSF examination to ensure any eosinophils are not mistaken for neutrophils.

The optimal treatment for Angiostrongylus meningoencephalitis remains poorly defined. Corticosteroids are commonly used, with the rationale of dampening the inflammatory reaction to the nematode, and have been 


\section{Case reports}

shown in a double-blind, placebo-controlled trial to provide symptomatic relief in eosinophilic meningitis. ${ }^{9}$ However, studies of patients with the encephalitic form of the disease have not found corticosteroids to be effective. ${ }^{3}$ Anthelmintics are generally not used due to the theoretical possibility of exacerbating cerebral inflammation and damage as a result of larval death in the central nervous system (CNS), and the lack of evidence of their efficacy. ${ }^{9,10}$ We used albendazole when there was little to lose and, perhaps as expected, it did not lead to any appreciable benefit. In the absence of effective treatment of angiostrongyliasis, it is important in endemic areas that the public understand the small but very serious risks associated with ingestion of uncooked molluscs.

It is known that time spent in a minimally conscious state following traumatic brain injury does not correlate with the chance of functional recovery. ${ }^{11}$ This observation may extend to patients with diffuse brain injury caused by severe cerebral infection or inflammation. This case shows the potential for the CNS to recover following a severe, generalised insult in a young patient with supportive care. It is important for doctors to appreciate this capacity when wrestling with difficult decisions about continuation of care for critically unwell patients.
Acknowledgements: We thank Dennis Hokin and Jennifer Posen at San Pathology, Sydney Adventist Hospital for assistance with histopathology.

Competing interests: No relevant disclosures.

Received 11 Jul 2012, accepted 31 Jan 2013.

1 Wang QP, Lai DH, Zhu XQ, et al. Human angiostrongyliasis. Lancet Infect Dis 2008; 8: 621-630.

2 Sawanyawisuth K, Takahashi K, Hoshuyama T, et al. Clinical factors predictive of encephalitis caused by Angiostrongylus cantonensis. Am J Trop Med Hyg 2009; 81: 698-701.

3 Chotmongkol V, Sawanyawisuth K. Clinical manifestations and outcome of patients with severe eosinophilic meningoencephalitis presumably caused by Angiostrongylus cantonensis. Southeast Asian J Trop Med Public Health 2002; 33: 231-234.

4 Prociv P, Carlisle MS. The spread of Angiostrongylus cantonensis in Australia. Southeast Asian J Trop Med Public Health 2001; 32 Suppl 2: 126-128.

5 Cooke-Yarborough CM, Kornberg AJ, Hogg GG, et al. A fatal case of angiostrongyliasis in an 11-month-old infant. Med J Aust 1999; 170: 541-543.

6 Prociv P. Parasitic meningitis. Med J Aust 1999; 170: 517-518.

7 Pryor DS, Konecny P, Senanayake SN, Walker J. First report of human angiostrongyliasis acquired in Sydney. Med J Aust 2003; 179: 430-431.

8 Diao Z, Xiao H, Wang J, et al. Identification of factors associated with clinically severe angiostrongyliasis. Am J Trop Med Hyg 2010; 83: 1352-1355.

9 Chotmongkol V, Sawanyawisuth K, Thavornpitak Y. Corticosteroid treatment of eosinophilic meningitis. Clin Infect Dis 2000; 31: 660-662.

10 Kliks MM, Kroenke K, Hardman JM. Eosinophilic radiculomyeloencephalitis: an angiostrongyliasis outbreak in American Samoa related to ingestion of Achatina fulica snails. Am J Trop Med Hyg 1982; 31: 1114-1122.

11 Lammi MH, Smith VH, Tate RL, Taylor CM. The minimally conscious state and recovery potential: a follow-up study 2 to 5 years after traumatic brain injury. Arch Phys Med Rehabil 2005; 86: 746-754. 